\title{
Erratum to: Large Scale Problems in Practice: The Effect of Dimensionality on the Interaction Among Variables
}

Fabio Caraffini, Ferrante Neri, and Giovanni Iacca

\author{
Erratum to: \\ Chapter "Large Scale Problems in Practice: \\ The Effect of Dimensionality on the Interaction Among \\ Variables" in: G. Squillero and K. Sim et al. (Eds.): \\ Applications of Evolutionary Computation, Part I, LNCS 10199, \\ https://doi.org/10.1007/978-3-319-55849-3_41
}

The original version of the paper starting on p. 636 was revised. An acknowledgement has been added. The original chapter was corrected.

The updated online version of this chapter can be found at https://doi.org/10.1007/978-3-319-55849-3_41 